\title{
Assessment of Quality of Life of Patients with Brain Tumors
}

\author{
Suela Kalaja ${ }^{1}$, Anita Pilika ${ }^{2}$, Artan Simaku ${ }^{3 *}$ \\ ${ }^{I}$ Neuroscience Hospital, University Hospital Centre "Mother Teresa", Tirana, Albania \\ ${ }^{2}$ Psychiatric Hospital Hospital, University Hospital Centre "Mother Teresa”, Tirana, Albania \\ ${ }^{3}$ Institute of Public Health, Tirana, Albania
}

*Corresponding Authors: Artan Simaku, Institute of Public Health, Tirana, Albania

\begin{abstract}
:
Introduction: Cancer as a disease and therapies administered for its treatment indicate that patients undergo major trauma, different from one person to another, and these individuals react differently. The aim of the study was to analyze the quality of life $(Q o L)$ and the level of depression anxiety and stress patients with brain tumours before and after psychotherapy.
\end{abstract}

Material and Methods: The instruments used in the study are: European Organization for Research and Treatment of Cancer Quality of Life Questionnaire 30 (EORTC QLQ-C30) and the questionnaire on the level of, Depression, Anxiety and Stress - the Albanian version for the patients with cancer. The study was conducted in 60 patients over the period 2015-2016 at the Department of Neuro-Oncology of the University Hospital Center "Mother Theresa" in Tirana. Comparative methodology has been used - individual intervention with involvement in the group. Presentation on case, case recording by physician, meeting and receiving information from family members were initial steps of the study.

Results: After the therapy, a significant improvement in the quality of life of patients was found in global score $(p<0.01)$ and functional scales: physical $(p=0.02)$, emotional $(p=0.03)$ and Symptoms scales: fatigue $(p=0.01)$, and constipation $(p<0.01)$. After the therapy, a significant improvement was found regarding the depression ( $p<0.01)$, anxiety $(p<0.01)$ and stress levels $(p<0.01)$ of patients.

Conclusion: The implementation of such an intervention means the creation of a multi-disciplinary and interdisciplinary approach in order to address the ill person in its entirety and thus to meet his / her needs.

Keywords: Cerebral Cancer, Patient, Psychotherapy, Quality of Life

\section{INTRODUCTION}

Recognition is growing that psychosocial care is an essential component of the comprehensive care of people diagnosed with cancer [1,2]. In addition to attempting to extend survival rates in people following a cancer diagnosis, the oncology community is recognizing the value of quality of life. Psychosocial care, with its goals of relieving emotional distress and promoting wellbeing, is central to efforts to improve quality of life. 2 However, evidence suggests that many patients with cancer who might benefit from psychosocial care do not receive it [2]. Primary brain tumours develop from brain cells and can be either benign (non-cancerous) or malignant (cancerous). Generally, benign tumours remain in the part of the brain in which they started, which can be problematic depending on the location, while malignant tumours can spread into the surrounding brain tissue. Both types of tumours can cause pressure and damage to the brain. The cause of most primary brain tumours is unknown. Secondary brain tumours can occur when cancer cells from other parts of the body spread to the brain. Many individuals are diagnosed with a malignant primary brain tumour each year. Although primary brain tumours account for less than 2 per cent of cancers, they can impose a large burden on individuals, families and carers [3,4]. Treatment usually involves surgery to remove as much of the tumour as possible, generally followed by radiotherapy and/or chemotherapy. Benign tumours can be as difficult to treat as malignant tumours, depending on their location within the brain and whether surrounding brain tissue could be damaged by surgery. Treatment for primary brain tumours may start very quickly, which means the person affected and his or her family and friends may have little time to come to terms with the diagnosis before treatment begins. There are many types of brain tumours 
and the likely outcome varies, depending on the type and grade of tumour. Many tumours are treatable, while others are controllable for years. Some tumours respond to treatment initially, but have a tendency to come back. Therefore, many people with brain tumours have to adapt to living with the effects of the tumour, its treatment and the threat of a recurrence [5,6]. While some people with a brain tumour make a complete recovery after treatment, most require some support and care in the following years, particularly from relatives and friends. The stress associated with giving this care can be considerable. HRQoL is a complex, multidimensional construct with a range of conceptual definitions. HRQoL may incorporate a wide variety of issues including general health, physical symptoms, functionality, toxicity, emotional well-being, cognitive issues, role functioning, social well-being, sexual functioning, existential/spiritual issues, financial status, job satisfaction and living conditions $[7,8]$. The patient is the best and most accurate judge of HRQoL at a particular point in time or over the course of an illness. HRQoL ratings by physicians have been found to differ significantly from the patient reports $[9,10]$. One of the most important aspects of baseline HRQoL is that it serves as an independent prognostic factor for survival or locoregional control. In some studies, it was found that HRQoL scores were related with survival. Moreover, baseline HRQoL scores were the strongest independent prognostic factor for survival or locoregional control in cancer patients [11]. The aim of the study was to analyze the QoL and the level of depression anxiety and stress patients with brain tumours before and after psychotherapy.

\section{MATERIAL AND MethodS}

The study was conducted in the period 2015-2016 at the Department of Neuro-Oncology of the University Hospital Center "Mother Theresa" in Tirana. Comparative methodology has been used individual intervention with involvement in the group. Presentation on case, case recording by physician, meeting and receiving information from family members were initial steps of the study. Three preoperative meetings with the patient were conducted and two with family members. 7-15 therapeutic sessions took place after surgery and 2-5 sessions as outpatient and the responsible caregiver. Psychotherapy sessions during the hospitalization were performed depending on the condition of the patient almost every day, 55 minutes - 1 hour, while after leaving the hospital 1-2 once a year as long as it was deemed necessary. The study was conducted with 120 hospitalized patients with cerebrovascular disease divided into two group. Group I - 60 patients performed individual therapy: Group II - 60 patients performed individual therapy and counseling sessions were provided for their family members (caregivers) $(n=60)$.

The instruments used in the study are: European Organization for Research and Treatment of Cancer Quality of Life Questionnaire 30 (EORTC QLQ-C30) and the questionnaire on the level of, Depression, Anxiety and Stress - the Albanian version for the patients with cancer. The implementation of such an intervention means the creation of a multi-disciplinary and interdisciplinary approach in order to address the ill person in its entirety and thus to meet his / her needs. We used the EORTC QLQ-C30 version 3.0, which is the most commonly used HRQoL instrument in cancer trials, is a 30- item cancer-specific questionnaire measuring general HRQoL in cancer patients $[12,13]$. The EORTC QLQC30 incorporates five functional scales (physical (PF), role (RF), emotional (EF), cognitive (CF) and social (SF) functioning scales), three symptom scales (fatigue, nausea/vomiting and pain), six single item scales (dispnea, insomnia, appetite loss, constipation, diarrhea and financial impact), and the overall health/global HRQoL scale. All items are scored on 4-point Likert scales ranging from 1 (not at all) to 4 (very much), except for the two items (items 29 and 30) in the overall health/global HRQoL subscale which were scored on a modified 7point linear analogue scale. All the raw functional scales and individual item scores were transformed to a linear scale that ranged from 0 to 100 , in which a higher score represented a higher level of functioning or an improved level of symptoms. The items were scaled and scored by using the recommended EORTC procedures [14]. EORTC C-30 scores were calculated by a computer-based program. DASS-42 (Depression, Anxiety and Stress) questionnaire was used to assess the level of depression anxiety and stress patients with brain tumours.

\section{Statistical Analysis}

Statistical analyses were made by SPSS software (version 13.0). Scores of the questionnaires were expressed as mean ( \pm standard deviation) and median, where appropriate. The scores were nonnormally distributed and were, therefore, compared by nonparametric methods. A $p<0.05$ was considered to be statistically significant. 


\section{RESULTS AND DISCUSSION}

The mean age of patients was 51 years (range: 11-75 years). Forty one (63.8\%) were male and 19 patients $(31.7 \%)$ were female. Baseline and after psychotherapy EORTC QLQ-C30 scores are shown in table1.

Table1. Comparison of score of QoL of patients before and after psychotherapy

\begin{tabular}{|c|c|c|c|}
\hline \multirow{2}{*}{ Dimensions } & Before Therapy & After Therapy & \multirow[t]{2}{*}{$\mathbf{P}$} \\
\hline & M (Sd) & M (Sd) & \\
\hline \multicolumn{4}{|c|}{ EORTC QLQ-C30 } \\
\hline Global score & $65.28 \pm 13.69$ & $71.37 \pm 17.42$ & $<0.01$ \\
\hline \multicolumn{4}{|c|}{ Functional Scales } \\
\hline Physical & $75.56 \pm 19.35$ & $81.47 \pm 19.43$ & 0.02 \\
\hline Role function & $77.78 \pm 20.51$ & $80.08 \pm 20$ & 0.1 \\
\hline Emotional & $70.83 \pm 26.23$ & $76.34 \pm 23.06$ & $\mathbf{0 . 0 3}$ \\
\hline Cognitive & $69.44 \pm 26.43$ & $70.76 \pm 23.86$ & 0.8 \\
\hline Social function & $68.06 \pm 27.02$ & $70.62 \pm 23.54$ & 0.4 \\
\hline \multicolumn{4}{|c|}{ Symptom Scales } \\
\hline Fatigue & $37.04 \pm 26.1$ & $30.79 \pm 21.32$ & 0.01 \\
\hline Nausea/vomiting & $4.17 \pm 10.36$ & $7.91 \pm 14.89$ & 0.1 \\
\hline Pain & $12.5 \pm 17.59$ & $12.57 \pm 14.11$ & 0.9 \\
\hline Dyspnea & $8.33 \pm 15.07$ & $7.34 \pm 15.18$ & 0.2 \\
\hline Insomnia & $27.78 \pm 27.83$ & $27.97 \pm 28.23$ & 0.8 \\
\hline Appetite loss & $19.44 \pm 17.16$ & $18.36 \pm 25.26$ & 0.7 \\
\hline Constipation & $5.56 \pm 19.25$ & $21.47 \pm 28.42$ & $<0.01$ \\
\hline Diarrhea & $3.24 \pm 12.74$ & $4.24 \pm 12.74$ & 0.8 \\
\hline Financial impact & $25 \pm 25.13$ & $22.03 \pm 26.59$ & 0.1 \\
\hline
\end{tabular}

After the therapy, a significant improvement in the quality of life of patients was found in global score $(\mathrm{p}<0.01)$ and functional scales: physical $(\mathrm{p}=0.02)$, emotional $(\mathrm{p}=0.03)$ and Symptoms scales: fatigue $(\mathrm{p}=0.01)$, and constipation $(\mathrm{p}<0.01)$. The level of depression, anxiety and stress before and after psychotherapy is shown in table 2 .

Table2. The level of depression, anxiety and stress before and after psychotherapy

\begin{tabular}{|c|c|c|c|}
\hline \multirow[t]{2}{*}{ Subscales } & Before Therapy & After Therapy & \multirow[b]{2}{*}{$\mathbf{p}$} \\
\hline & M (SD) & M (SD) & \\
\hline Depression & $29.1(9.9)$ & $12.1(3.9)$ & $<0.01$ \\
\hline Anxiety & $27.3(11.3)$ & $14.9(6.8)$ & $<0.01$ \\
\hline Stress & $32.0(14.1)$ & $16.8(7.9)$ & $<0.01$ \\
\hline Total scale & $88.4(35.3)$ & $43.8(18.6)$ & $<0.01$ \\
\hline
\end{tabular}

After the therapy, a significant improvement was found regarding the depression $(\mathrm{p}<0.01)$, anxiety $(\mathrm{p}<0.01)$ and stress levels $(\mathrm{p}<0.01)$ of patients.

\subsection{Discussion}

Brain tumours can cause a variety of symptoms. These can take the form of neurologic symptoms (e.g. headaches, dizziness, impairment of speech or movement), cognitive changes (e.g. memory problems) and behavioural changes (e.g. changes in the way people think or act). These effects can be due to the brain tumour itself, or to surgery, medication (such as steroids which are commonly used to reduce swelling in the brain), or other treatments such as radiotherapy or chemotherapy [15].. Changes can be short-term or lifelong, may get better or worse over time. Experiencing any of these symptoms can be distressing and frustrating for the person with the brain tumour and for their family members and carers. Experiencing any of these symptoms can be distressing and frustrating for the person with the brain tumour and for their family members and carers. Anxiety is more than just feeling stressed or worried. Anxious feelings are a normal reaction to a situation where a person feels under pressure and usually pass once the stressful situation has passed, or 'stressor' is removed. However, for some people these anxious feelings happen for no apparent reason or continue after the stressful event has passed. For a person experiencing anxiety, anxious feelings cannot be brought under control easily [16]. Anxiety can be a serious condition that makes it hard for a person to cope with daily life. There are many types of anxiety and many people with anxiety experience symptoms 
of more than one type. Living with a brain tumour is one of many things - such as a family history of mental health conditions, stressful life events and personality factors - that may trigger anxiety. The combination of chronic physical illness, lost educational or employment opportunities and financial worries can lead to the development of anxiety. Anxiety is common and the sooner a person gets help, the sooner they can recover $[17,18]$.

The symptoms of anxiety can often develop gradually over time. Given that we all experience some anxiety, it can be hard to know how much is too much. In order to be diagnosed with an anxiety condition, it must have a disabling impact on the person's life. Anxiety can be expressed in different ways such as uncontrollable worry, intense fear (phobias or panic attacks), upsetting dreams or flashbacks of a traumatic event. While we all feel sad, moody or low from time to time, some people experience these feelings intensely, for long periods of time (weeks, months or even years) and sometimes without any apparent reason. Depression is more than just a low mood - it's a serious condition that has an impact on both physical and mental health. Depression affects how a person feels about themselves. A person may lose interest in work, hobbies and doing things he or she normally enjoys. Some people may lack energy, have difficulty sleeping or sleep more than usual, while some people feel anxious or irritable and find it hard to concentrate. A person may be depressed if he or she has felt sad, down or miserable most of the time for more than two weeks and/or has lost interest or pleasure in usual activities, and has also experienced some of the signs and symptoms on the list below. Commonly, shock, sadness and anger can last for up to a month after the diagnosis of a brain tumour [19]. Unless symptoms are very severe, doctors normally would not diagnose depression at this time. It is important to note, however, that people will work through the various emotions associated with being diagnosed with a brain tumour in their own time. Even after a person comes to terms with having a brain tumour, he or she is at increased risk of depression from the mental and physical effects of the tumour, side-effects of treatments, and from reduced quality of life [20]..

Studies report depression rates in people with brain tumours range from 15 per cent to 38 per cent, although it is suggested these rates are likely to under-represent the true incidence. A survey of people with brain tumours found that 13 per cent were severely or extremely stressed and 23 per cent were severely or extremely anxious.3 It's important to note that stress is not depression, however, acute distress can occur and may be a risk factor for depression if it persists. Depression in people with a brain tumour may also be closely linked to emotional distress.

Levels of depression may increase over time. Several factors can greatly increase levels of stress and anxiety in a person with a brain tumour: the diagnosis of a brain tumour may cause emotions such as shock and disbelief, despair, anger and anxiety, helplessness, loss and worry about dying. These are all a normal part of adapting to this kind of major life event and the way people view themselves, their lives and the future. However, for some people, these feelings do not pass or ease with time and can lead to anxiety or depression. The physical effects of a brain tumour (e.g. seizures, restricted movement or functioning) can limit a person's independence (such as the ability to drive a car) and quality of life $[21,22]$. This can result in difficulties socially, at work and in everyday life. The continuing strain associated with living with a brain tumour, and feelings of guilt about the strain placed on family members and others, together with the side-effects of treatment can further increase levels of stress and anxiety. Mild anxiety or depression, through to psychological and medical treatment for more moderate to severe conditions. Support groups may also be helpful for people with a brain tumour, their families and carers. If there is concern about behavioural changes and their effect on a person's quality of life, it's important to seek help as soon as possible. A doctor can help the person minimise the effects of the changes and decide whether treatment might be needed. Psychological treatments: Psychological therapies may not only help with recovery, but can also help prevent a recurrence of anxiety or depression. These therapies help build skills in coping with stressful life circumstances and can be provided by a psychologist, psychiatrist or other trained health professional. Cognitive behaviour therapy (CBT) is an effective treatment for people with anxiety and depression. It teaches people to evaluate their thinking about common difficulties, helping them to change their thought patterns and the way they react to certain situations. Interpersonal therapy (IPT) is also effective for treating depression and some types of anxiety. It helps people find new ways to get along with others and to resolve losses, changes and conflict in relationships [23,24]. Sometimes, people with brain tumours have difficulty with language - either in understanding or expressing. In such instances, psychological therapies such as CBT and IPT may prove difficult. 
Depending on the side-effects caused by the brain tumour, the health professional should be able to tailor treatment to a person's particular needs. Making a decision about which antidepressant is best for a person can be complex. The decision will be made in consultation with a doctor, after careful assessment and consideration. The doctor will make an overall judgment about the suitability of any treatment based on several factors - including the person's clinical condition, other treatments the person is having, the effectiveness of any previous antidepressant treatment and the person's own preferences. The doctor should discuss differences in effects and possible side-effects of medications. Stopping medication should only be done gradually, with a doctor's recommendation and under supervision. Regular contact with and ongoing assessment by a doctor to check that treatments are working effectively is an important part of becoming and staying well. Most people taking medication will also benefit from psychological therapies, which will reduce the likelihood of relapse after the person has stopped taking the medication. Managing anxiety and derpression can greatly improve people's wellbeing and quality of life. People with anxiety and/or depression can find it difficult to take the first step in seeking help. They may need the support of family, friends and a health professional. There is no one proven way that people recover from anxiety or depression and it's different for everybody. However, there is a range of effective treatments and health professionals who can help people on the road to recovery $[25,26]$. There are also many things that people with anxiety and depression can do to help themselves to recover and stay well. The important thing is finding the right treatment and the right health professional that works for you. Different types of anxiety and depression require different types of treatment. This may include physical exercise for preventing and treating [27].

Antidepressant medication, alongside psychological therapies, can also play a role in the treatment of moderate to severe depression and some anxiety conditions. No studies have yet examined the effectiveness of antidepressants specifically in people with brain tumours, but they have been found to be effective in the wider adult population. The clinical implications of this study are clear: QOL is often poor in brain tumor patients, and could potentially be improved if depression can be identified and managed. According to our results, it appears that the presence of depression should be investigated in every brain tumor patient, and if it is identified, it should be treated as early as possible. Depression may be closely correlated with fatigue, emotional distress, and unaddressed existential issues, and specific interventions for each of these problems are often readily available. Antidepressant medications and psychotherapy (particularly cognitive behavior therapy) have been shown to be of comparable effectiveness in the treatment of major depression, although the studies available so far have been conducted mostly with populations from which major depression with psychotic features or problematic medical conditions were excluded [28-30]. Our knowledge of the effectiveness of psychotherapy for depression in brain tumor patients or other neurologically impaired populations remains limited, but cognitive behavior therapy has been identified as worthy of additional investigation[31]. Also, participation in support groups proved to be helpful for many patients and their partners [32]. Although for many brain tumor patients, treating depression with psychotherapy may present a welcome relief from the use of medications, those who also experience significant fatigue or who may have limitations preventing them from benefiting from psychotherapy may do better with antidepressant medication or with psychostimulants such as methyphenidate [33] similarly, brain tumor patients may benefit from recently developed pastoral or psychotherapeutic interventions designed to help people come to terms with existential and spiritual concerns [34].

\section{CONCLUSION}

More research is needed to acquire a better understanding of the correlates of depression and existential tension in brain tumor patients, with the view of arriving eventually at treatments that could be more specifically tailored for this population. Other QOL issues, such as sense of control over treatment, the use of alternative/complementary therapies, and the relationship between the QOL of caregivers and the QOL of patients need to be explored. Finally, it is becoming increasingly obvious that the assessment and treatment of patients' psychosocial issues such as emotional distress, fatigue, and existential problems need to be integrated to the medical treatment in order to provide optimum care to brain tumor patients.

\section{REFERENCES}

[1] Mauer ME, Bottomley A, Taphoorn MJ et al. Evaluating health-related quality of life and symptom burden in brain tumour patients: instruments for use in experimental trials and clinical practice. Curr Opin Neurol 2008; 21:745-753. 
[2] Taphoorn MJB, Klein M. Cognitive deficits in adult patients with brain tumors. Lancet Neurol 2004; 3:159-168.

[3] Hahn CA, Dunn RH, Logue PE et al. Prospective study of neuropsychologic testing and quality-of-life assessment of adults with primary malignant brain tumors. Int J Radiat Oncol Biol Phys. 2003 Mar 15;55(4):992-9.

[4] Parvataneni R, Polley MY, Freeman F et al. Identifying the needs of brain tumor patients and their caregivers. J Neurooncol. 2011;104(3):737-44.

[5] Strang S, Strang P, Ternestedt BM. Existential support in brain tumour patients and their spouses. Support Care Cancer. 2001;9(8):625-33.

[6] Madsen K, Poulsen HS. Needs for everyday life support for brain tumour patients' relatives: systematic literature review. Eur J Cancer Care (Engl). 2011;20(1):33-43.

[7] Salander P, Spetz A. How do patients and spouses deal with the serious facts of malignant glioma? Palliat Med. 2002;16(4):305-13.

[8] Pitceathly C, Maguire P. The psychological impact of cancer on patients' partners and other key relatives: A review. European Journal of Cancer 2003;39:1517-1524.

[9] Wong RKS, Franssen E, Szumacher E. What do patients living wiht advanced caner and their carers want to konw? - A needs assessment. Support Care Cancer. 2002;10:408-15.

[10] Cavers D, Hacking B, Erridge SE et al. Psychological and existential well-being in patients with glioma and their caregivers: a qualitative study. CMAJ. 2012 Mar 19.

[11] Bottomley A, Vanvoorden V, Flechtner H et al. The challenge and achievements of implementation of quality of life research in cancer clinical trials. Eur J Cancer 2003; 39:275-285.

[12] Faller H, Schuler M, Richard M, Heckl U, Weis J, Kuffner R. Effects of psychooncologic interventions on emotional distress and quality of life in adults patients with cancer: systematic review and meta-analysis. $J$ Clin Oncol (2013) 31:782-93. doi:10.1200/JCO.2011.40.8922

[13] Aaronson NK, Ahmedzai S, Bergman B, Bullinger M, Cull A, Duez NJ (1993) The European Organization for Research and Treatment of Cancer EORTC C30: a quality of life instrument for use in international clinical trials in oncology. J Natl CancerInst 85:365-376

[14] Fayers PM, Aaronson NK, Bjordal K, Groenvold M, Curan D, Bottomley A, on behalf of the EORTC Quality of Life Study Group (2001) The EORTC QLQ-C30 scoring manual, 3rd edn. European Organization for Research and Treatment of Cancer,Brussels

[15] Klein M, Taphoorn MJB, Heimans JJ et al. Neurobehavioral status and health-related quality of life in newly diagnosed high-grade glioma patients. J Clin Oncol 2001; 19:4037-4047.

[16] Wen PY, Schiff D, Kesari S et al. Medical management of patients with brain tumors. J Neurooncol 2006; 80: 313-32.

[17] Mauer ME, Bottomley A, Taphoorn MJ et al. Evaluating health-related quality of life and symptom burden in brain tumour patients: instruments for use in experimental trials and clinical practice. Curr Opin Neurol 2008; 21:745-753.

[18] Flechl B, Ackerl M, Sax C et al. Neurocognitive and sociodemographic functioning of glioblastoma longterm survivors. J Neurooncol 2012 Sep;109(2):331-9.

[19] Henriksson R, Asklund T, Poulsen HS: Impact of therapy on quality of life, neurocognitive function and their correlates in glioblastoma multiforme: e review. J Neuroonocol 2011; 104:639-646.

[20] Giovagnoli AR: Investigation of cognitive impairments in people with brain tumors. J Neurooncol. 2012 Jun;108(2):277-83

[21] Mauer M, Stupp R, Taphoorn MJ et al. The prognostic value of health-related quality-of-life data in predicting survival in glioblastoma cancer patients: results from a an international randomized phase III EORTC Brain Tumour and Radiation Oncology Groups, and NCIC Clinical Trials Group study: British J of Cancer 2007; 97:302-307.

[22] Liu R, Page M, Solheim K et al. Quality of life in adults with brain tumors: current knowledge and future directions. Neuro Oncol. 2009 Jun;11(3):330-9.

[23] Whiting DL, Simpson GK, Koh E-S, Wright KM, Simpson T, Firth R. A multitieredintervention to address behavioural and cognitive changes after diagnosisof primary brain tumour: a feasibility study. Brain Inj (2012) 26:950-61. doi:10.3109/02699052.2012.661912

[24] Ross DA, Cetas JS. Steroid psychosis: a review for neurosurgeons. J Neurooncol. 2012;109(3):439-447.

[25] Kolthof HJ. Moderne antidepressiva en hallucinaties. Tijdschr Psychiatr. 2014;56(6):407-412.

[26] Sivec HJ, Montesano VL. Cognitive behavioral therapy for psychosis in clinical practice. Psychotherapy. 2012;49(2):258. 
[27] Chadwick P. Mindfulness for psychosis. Br J Psychiatry. 2014;204(5):333-334.

[28] White R, Gumley A, McTaggart J, et al. A feasibility study of acceptance and commitment therapy for emotional dysfunction following psychosis. Behav Res Ther. 2011;49(12):901-907.

[29] Thomas N, Hayward M, Peters E, et al. Psychological therapies for auditory hallucinations (voices): current status and key directions for future research. Schizophr Bull. 2014;40(suppl 4):S202-S212.

[30] Arnold, SD. Forman, LM. Brigidi, BD. Evaluation and characterization of generalized anxiety and depression in patients with primary brain tumors. Neuro-Oncology. 2008;10:171-181.

[31] Zarghi A, Zali A, Ashrafi F, Moazezi M. Neuroscience and Neuro-cognitive Rehabilitation. Basic Research Journal of Medicine and Clinical Sciences. 2013a;2(8),83-87.

[32] D’Angelo C, Mirijello A, Leggio L, Ferrulli A, Carotenuto V, Icolaro N, et al. Stateand trait anxiety and depression in patients with primary brain tumors before and after surgery: 1-year longitudinal study. J Neurosurg (2008) 108:281-6. doi:10.3171/JNS/2008/108/2/0281

[33] Goebel S, Strenge H, Mehdorn HM. Acute stress in patients with brain cancer during primary care. Support Care Cancer (2012) 20:1425-34. doi:10.1007/s00520-011-1225-6

[34] Kangas M, Williams JR, Smee RI. The association between post-traumatic stress and health-related quality of life in adults treated for a benign meningioma. Appl Res Qual Life (2012) 7:163-82. doi:10.1007/s11482-011-9159-1

Citation: Artan Simaku et. al. "Assessment of Quality of Life of Patients with Brain Tumors" International Journal of Humanities Social Sciences and Education (IJHSSE), vol 5, no. 8, 2018, pp. 92-98. doi: http://dx.doi.org/10. $20431 / 2349-0381.0508011$.

Copyright: (c) 2018 Authors. This is an open-access article distributed under the terms of the Creative Commons Attribution License, which permits unrestricted use, distribution, and reproduction in any medium, provided the original author and source are credited. 